\title{
Displaying digital holograms of real-world objects on a mobile device using tilt-based interaction
}

\author{
Taina M. Lehtimäki, Kirsti Sääskilahti, Marcin Kowiel \\ Oulu Southern Institute \\ University of Oulu \\ 84100 Ylivieska, Finland
}

\author{
Thomas J. Naughton \\ Department of Computer Science \\ National University of Ireland, Maynooth, Ireland, and \\ Oulu Southern Institute, University of Oulu \\ 84100 Ylivieska, Finland \\ tomn@cs.nuim.ie
}

\begin{abstract}
Holography is a well-known technique for sensing and displaying real-world three-dimensional (3D) objects. Reconstructions from digital holograms are typically displayed with regular two-dimensional (2D) screens and therefore lot of the 3D specific information is not passed on to the viewer during the display process. Mobile devices have interesting possibilities for displaying 3D data interactively. In this study, we show how nine viewers evaluated hologram reconstructions with a tilt based display system incorporated into a mobile device.
\end{abstract}

\section{INTRODUCTION}

Digital holography is a technique that gives the possibility to record and display 3D information of real-world objects. In digital holography $[1,2,3]$ a $3 \mathrm{D}$ scene is captured optically and the desired perspectives are reconstructed numerically with a computer. The reconstructions are routinely displayed in the form of a $2 \mathrm{D}$ image slice through the reconstruction volume, an extended focus image, or a depth map from a single perspective. These are not certain to give the human viewer a clear perception of the 3D scene encoded in the hologram.

Holograms have also been successfully displayed on stereoscopic displays allowing a human viewer to perceive some 3D information of the scene encoded in the hologram by displaying reconstruction from a different perspective of the same hologram to each of the left and right eyes of the viewer. Previously, we have reported results suggesting that when digital hologram reconstructions are displayed stereoscopically people perceive less noise, increased depth of field, better visibility of details, and better perceived quality when compared to displaying the reconstructions as $2 \mathrm{D}$ images $[4,5$, 6]. Also, a previous study has evaluated how viewers perceive depth in a single-view video reconstruction from a holographic dynamic 3D scene [7].

Displaying stereoscopically reconstructed images from holographic data, and also in the case of video, involves combining information from more than one reconstructed 2D image in the human visual system. We propose an interactive way of displaying 2D reconstructions fast enough to viewer so that their visual system would interpret the displayed set of images as single $3 \mathrm{D}$ object viewed from different perspectives.
An interesting possibility is to use a mobile device to present $3 \mathrm{D}$ data on the display. Devices with six degrees of freedom (DOF) input can offer an interactive way to perceive $3 \mathrm{D}$ objects by enabling the user to see different perspectives by moving device in different directions (tilt, rotate, and yaw) [8]. Mobile devices with a tilt-based user interface have been successfully demonstrated, for example for 3D geographic data visualization and for 3D virtual environment applications [9]. The idea of using tilt as an interaction technique to see a $3 \mathrm{D}$ object model on the small display has been proposed [10], but so far presentations of single real-world 3D objects are quite unknown to the research area.

The typical restrictions on a mobile device for displaying complex information are: the small display size, poor resolution, a small amount of memory, and low computation ability. These restrictions have motivated new interaction and information presentation methods [11]. For example, the development of motion sensing technologies allows the manipulation of onscreen content in real time, and thus some restrictions related to the small screen size can be alleviated.

One typical obstacle to a good quality display of digital hologram data is the noise in reconstructions which is present especially in reconstructions taken with a small window size. Also, the processing requirements for creating real-time reconstructions $[12,13]$ may restrict displaying of holograms on mobile devices until they incorporate dedicated graphics hardware comparable to that currently in today's laptop computers.

The goal of our study is to investigate if it is possible to support the 3D perception of digital holographic data captured of real-world objects by utilizing an interactive display system with tilt functionality. We present reconstructed views of the objects on the display of a mobile device with a tilt sensor. Our challenges related to small display size, large amount of noise in the reconstructions, and small amount of processing power. More precisely: (1) how to display noisy reconstructions on the small screen so that the viewers can still clearly perceive the object itself, and (2) how to display reconstructions from a new perspective fast enough so that the viewer would perceive a smoothly turning object but still sufficiently slow that details are still clearly visible. 
In the results and conclusions section we explain how we overcame the challenges listed above. We also show the results of how a group of viewers evaluated the disturbance of noise in two holograms displayed separately with and without tilt. With evaluations, we explain how the tilting method affects the amount of noise perceived. First, we explain the experimental methods in more detail.

\section{METHOD}

\section{A. Subjects and apparatus}

Nine subjects (2 male, 7 female) participated in this experiment. All participants were familiar with PDA (mobile personal digital assistant) devices and three of them had some experience using tilting as an interaction method.

We used a DELL Axim x51v (480x640 pixels screen, 624 $\mathrm{MHz}$ processor, Intel $\mathrm{G} 2700 \mathrm{G}$ graphics processor with $16 \mathrm{MB}$ of RAM) running Windows Mobile 5.0 as our mobile platform. This was particularly chosen for the good processing power and reasonable resolution display in comparison with alternatives available commercially. The PDA was combined with a TiltCONTROL tilt sensor from EcerTech Ltd. (Queensland, Australia), with 180 degrees vertical and 180 degrees horizontal sensing.

\section{B. Software}

Because of the limited processing power on the mobile device we decided to create the reconstructions of the hologram offline on a computer and store the resulting images on the mobile device for this experiment. However, it is envisaged that after a few years these computations could be performed on a later generation of the mobile device.

We wrote software which displays 36 reconstructed images from the hologram with perspective change 10 degrees between each other while tilting the device vertically to the left and to the right. This type of tilting imitates how person would turn an actual object on their hand when viewing it i.e. we used the metaphor of realistically rotating object to the left while tilting to left and at the same way to the right.

\section{Stimuli}

The holograms were captured of real-world objects, with side lengths of approximately $1 \mathrm{~cm}$, and the object images reconstructed numerically using simulated Fresnel propagation [14]. Mounting the object on a rotation stage allowed us to capture different sides of the object. The image sets used for this experiment contained 36 images reconstructed with vertical perspective changes of approximately 10 degrees between images (encompassing the 360 degree range of perspectives associated with one axis of rotation). The complete stimuli image set was created of two holograms and an example reconstruction of each is presented in Figure 1. The size of the displayed reconstructed images was $240 \times 240$ pixels. The original holograms were $2048 \times 2048$ pixels and we used $512 \times 512$ pixels window size to get different perspectives for our reconstructions.
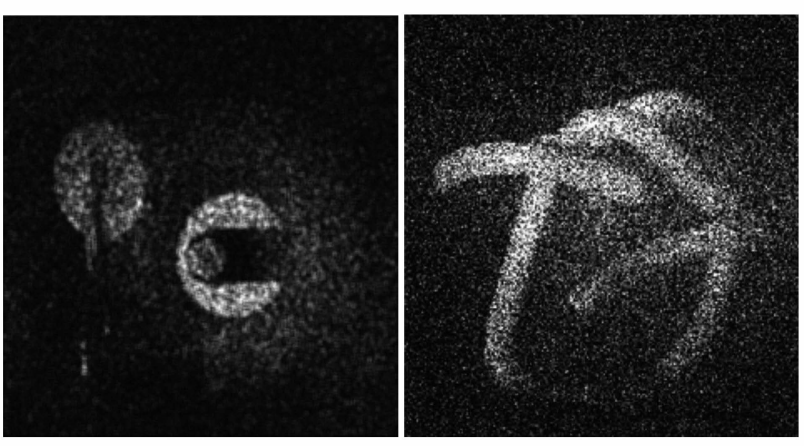

Figure 1. Objects TwoScrews (left) and Wires (right).

\section{Procedure}

Participants were advised to hold the device comfortably in their hand and keep the device at a constant distance from themselves as much as possible during the experiments. They were advised not to turn their head left or right while tilting the device for viewing the images.

The first task for the participants involved choosing a preferred relationship between tilt angle and induced perspective change on the display. The user selected their preference for one of the following three alternatives:

1. Tilting the device 10 degrees rotates the perspective of the object by 10 degrees.

2. Tilting the device 5 degrees rotates the perspective of the object by 10 degrees.

3. Tilting the device 15 degrees rotates the perspective of the object by 10 degrees.

After this selection the participants were asked to evaluate subjectively how much the noise disturbed the visual perception of the object without tilt functionality (i.e. a still 2D reconstruction of the object from one perspective) and with tilt functionality (where the displayed perspective of the object changes automatically in response to tilt). They were asked to use a scale from 1 to 7 (where 1 indicates that it disturbs very little, and 7 indicates that it disturbs significantly).

The participants were also asked to subjectively comment how well the tilting functionality in general supported the perception of objects in $3 \mathrm{D}$ in their opinion.

\section{RESULTS AND CONCLUSIONS}

Subjects reported that the interactive tilting very an interesting and pleasant way to observe the object with changing perspectives. Subjects clearly (eight out of nine) preferred alternative 2 when tilting the device $(5$ degrees changes the perspective of the object by 10 degrees). One subject preferred alternative 1 and none preferred alternative 3 . We may conclude that with this parameter, interactive tilting can be used to update new perspectives rapidly enough so that the viewers were able to perceive a smoothly turning object while ensuring that details were still visible. 
We were able to display the noisy reconstructions on the small screen so that the viewers were still able to perceive the object itself. Evaluations showed that using tilt indeed decreased the disturbance of noise, as documented in Figure 2. We may conclude that a tilting type of interactive displaying of different perspectives can be used to decrease the effect of noise for visual perception of the object. We believe that the inherent physiological and cognitive processes in the viewer performed the necessary temporal integration to filter out some of the noise.

The participants' subjective comments about the use of tilting to support their 3D perception of the object suggested that it is generally a good tool for this purpose. We may conclude that this type of interactive display method can support 3D perception of real-world three-dimensional (3D) objects captured by digital holography.

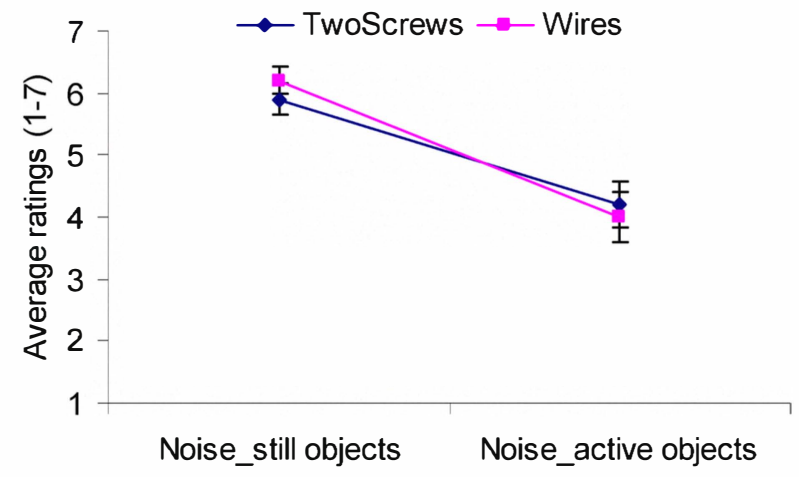

Figure 2. Subjectively evaluated disturbance of noise for visual perception of hologram reconstructions. On vertical axis average rating of nine viewers for disturbance of noise is shown, from 1 , disturbs very little, to 7 , disturbs very much). On horizontal axis is Noise_still objects evaluation without tilt functionality and Noise_active objects evaluation with tilt functionality.

\section{ACKNOWLEDGEMENTS}

The research leading to these results has received funding from Science Foundation Ireland under the National Development Plan, the Academy of Finland, and the European Community's Seventh Framework Programme FP7/2007-2013 under Grant agreement No. 216105 (acronym "Real 3D").
Thanks to Conor Mc Elhinney and Tomi Pitkäaho for assistance with the hologram capture and preparation.

\section{REFERENCES}

[1] D. Gabor, "A new microscopic principle," Nature, vol. 161, pp. 777-778, 1948.

[2] Th. Kreis, Handbook of Holographic Interferometry: Optical and Digital Methods, Wiley, 2005.

[3] Y. Frauel, T.J. Naughton, O. Matoba, E. Tajahuerce, and B. Javidi, "Three-dimensional imaging and processing using computational holographic imaging", Proceedings of the IEEE, vol. 94, pp. 636-653, 2006.

[4] T.M. Lehtimäki and T.J. Naughton, "Stereoscopic viewing of digital holograms of real-world objects", 3DTV-Conference 2007 - Capture, Transmission and Display of $3 D$ Video, article no. 39, Kos, Greece, 7-9 May 2007.

[5] T.M. Lehtimäki, K. Sääskilahti, R. Näsänen, and T.J. Naughton, "Visual perception of digital holograms on autostereoscopic displays". Proc. SPIE 7329, art. no. 73290C, 2009.

[6] T.M. Lehtimäki, K. Sääskilahti, R. Näsänen, and T.J. Naughton., "Stereo perception of reconstructions of digital holograms of real-world objects" Phys.: Conf. Ser. 206, article no. 012030, 2010.

[7] M. Paturzo, P. Memmolo, A. Finizio, R. Näsänen, T.J. Naughton, and P. Ferraro, "Synthesis and display of dynamic holographic 3D scenes with real-world objects," Optics Express, vol. 18, no. 9, pp. 8806-8815, 2010.

[8] A. Henrysson and M. Billinghurst, "Using a Mobile Phone for 6 DOF Mesh Editing," Proceedings of the 7th ACM SIGCHI New Zealand chapter's international conference on Computer-human interaction: design centered HCI, pp. 9-16, 2007.

[9] L. Baillie, H. Kunczier, and H. Anegg, "Rolling, Rotating and Imagining in a Virtual Mobile World," Proceedings of the 7th international conference on Human computer interaction with mobile devices \& services, pp. 283-286, 2005.

[10] J. Rekimoto, "Tilting operation for small screen interfaces," Proceedings of the 9th annual ACM symposium on User interface software and technology, pp. 167-168, 1996.

[11] L. Qiao, L. Feng, and L. Zhou. "Information presentation on mobile devices: techniques and practices," Lecture Notes in Computer Science vol. 4976, pp. 395-406, 2008.

[12] L. Ahrenberg, A. J. Page, B. M. Hennelly, J. B. McDonald, and T. J. Naughton, "Using commodity graphics hardware for real-time digital hologram view-reconstruction," Journal of Display Technology, vol. 5, pp. 111-119, 2009.

[13] H. Kang, F. Yaraş, and L. Onural, "Graphics processing unit accelerated computation of digital holograms," Applied Optics, vol. 48, pp. H137H143, 2009.

[14] C. P. Mc Elhinney, B. M. Hennelly, and T. J. Naughton, "Extended focused imaging for digital holograms of macroscopic three-dimensional objects," Applied Optics, vol. 47, pp. D71-D79, 2008. 